International Journal of Pure and Applied Mathematics

Volume 102 No. 2 2015, 247-263

ISSN: 1311-8080 (printed version); ISSN: 1314-3395 (on-line version)

url: http://www.ijpam.eu

doi: http://dx.doi.org/10.12732/ijpam.v102i2.7

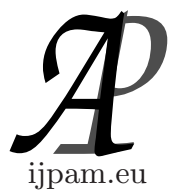

\title{
ANALYSIS ON THE EFFECTS OF WALL PROPERTIES ON MHD PERISTALTIC FLOW OF A DUSTY FLUID THROUGH A POROUS MEDIUM
}

\author{
Siva Parthasarathy ${ }^{1}$, Govindarajan Arunachalam ${ }^{2}$, M. Vidhya $^{3}$ \\ ${ }^{1,2}$ Department of Mathematics \\ SRM University \\ Kattankulathur, 603203, INDIA \\ ${ }^{3}$ Department of Mathematics \\ Sathyabama University \\ Sholinganallur, 603203, INDIA
}

\begin{abstract}
In this paper, a MHD peristaltic transport of dusty fluid through a porous medium in a uniform channel with elastic wall properties has been considered. This phenomenon is modeled on a system of partial differential equations governing the motion of the fluid and solid particles. This system of Navier stokes equations is linearized under long wavelength approximation and solved with appropriate boundary conditions using perturbation technique. The analytical solution is obtained for streamline functions and axial velocity profiles for both fluid and dust particles. The effects of membrane tension parameter, mass characterizing parameter, damping parameter, mass concentration of the dust particles, Hartmann number and permeability of the porous medium on streamline pattern of the fluid is studied. It is observed that the trapped bolus size increases with the increase in tension parameter, Hartmann number and permeability of the porous medium, but decreases with increase in damping and mass concentration parameters.
\end{abstract}

Received: February 20, 2015

(C) 2015 Academic Publications, Ltd. url: www.acadpubl.eu

$\S_{\text {Correspondence author }}$ 
AMS Subject Classification: 76S05, 76T20, 74F10, 35K41

Key Words: wall properties, MHD, peristaltic flow, porous medium, trapping, reflux

\section{Introduction}

Peristalsis is the movement of body fluids travelling through tubular organs continuously by contraction and relaxation due to change in pressure along the walls. This phenomenon is observed in the human body during the transport of lymph on lymphatic vessels, while swallowing food through the esophagus to the stomach, the vasomotion of small blood vessels, transport of chyme through the small intestine, the passage of urine from the kidneys through the urethra to the urinary bladder, the flow of semen in the male reproductive tract, and the movement of ovum in the female fallopian tube. Peristaltic phenomenon is used in biomedical instruments like dialysis machines, heart-lung machines, artificial heart and ortho machines, and transport of toxic material waste inside the sanitary ducts. Peristaltic concept is used in nuclear industry to avoid contamination of the outside environment.

Beginning with the first investigation of Latham [2], several theoretical and experimental attempts have been made to understand peristaltic action in different situations. The theory of trapping and reflux was first developed by Shapiro et al. [3]. Shapiro discussed the theoretical results for both plane and axisymmetric geometries. Srinivasa Rao [19] studied the peristaltic transport of non uniform channel under the long wavelength approximation. Muthu et al. [8] studied the influence of wall properties in the peristaltic motion of a micropolar fluid. Mochtar et al. [9] developed a new model to study the effect of wall properties on peristaltic transport of a viscous fluid. Influence of wall properties on peristaltic transport with heat transfer was studied by Radhakrishnamacharya et.al [11]. Hina et al. [17] discussed the effects of wall properties on peristaltic motion with respect to heat/mass transfer. Muthu et al. [12] considered peristaltic motion of micropolar fluid in circular cylindrical tubes. Sankad et al. [14] analyzed the effect of wall properties on peristaltic transport of micropolar fluid under long wavelength approximation. Few others have discussed the trapping and reflux for different kind of fluids. Hayat et al. [16] analyzed the effect of wall properties on the peristaltic flow of a third grade fluid in a curved channel with heat and mass transfer. Dheja et al. [20], Nadeem et al. [21] studied the trapping and reflux for Jeffery fluid. Sankad et al. [23] have discussed the peristaltic flow of Hershel- Bulkley fluid. The MHD flow of a 
fluid in a channel with elastic rhythmically contracting walls (peristaltic flow) is of interest in connection with certain problems of the movement of conductive physiological fluids, and with the need for theoretical research on the operation of a peristaltic MHD compressor. Also the principle of magnetic field may be used in clinical application (magnetic resonance imaging MRI). Makhimer et al. [7] discussed the nonlinear peristaltic transport of Magneto-hydrodynamics (MHD) flow through a porous medium. Effects of magnetic field and wall properties of micropolar fluid on peristaltic flow was discussed by Afifia et al. [15]. Moreover, recently Dheia et al. [20] studied the effects of wall properties and heat transfer on the peristaltic flow of Jeffry fluid through porous medium. The two-phase flow plays an important role in recent times in the purification of oil in the oil industry. Also, such flows occur in fluidization flow in rocket tubes, blood flow in capillaries, paint spraying confusion etc. Saffmans [1] dusty fluid model serves as a good model for describing blood as a binary system. Tridib et al. [4] investigated the influence of wall properties and Poiseuille flow in peristalsis. Kaimal [5], Radhakrishnamacharya [6] studied peristaltic transport of a solid-fluid mixture through a two dimensional channel. Mohammed Haroun [10] detailed the effect of wall compliance on peristaltic transport of a Newtonian fluid in an asymmetric channel. Srinivasacharya [13] studied the effects of wall properties on peristaltic transport of a dusty fluid and explained trapping and reflux in their article. Nabil Eldabe et al. [18] discussed the wall properties of peristaltic motion with heat and mass transfer of a dusty fluid through a horizontal porous channel. The peristaltic flow of viscous fluid in a rectangular duct with compliant walls has been studied by Nadeem et al. [22].

The primary goal of this article is to construct a mathematical model to examine the influence of wall properties on unsteady MHD peristaltic flow of a dusty fluid through the porous medium in a symmetric channel. This phenomenon is modeled on a system of partial differential equations governing the motion of fluid and solid particles. Problem is formulated in Section 2. Section 3 includes the solutions for the problem by using perturbation method. Numerical results and discussion are given in Section 4. The conclusions have been summarized in Section 5 .

\section{Formulation of the Problem}

We consider the peristaltic transport of an incompressible fluid that contains small solid particles, whose number density $\mathrm{N}$ (assumed to be constant) is large enough to define average properties of the dust particles at a point through a 


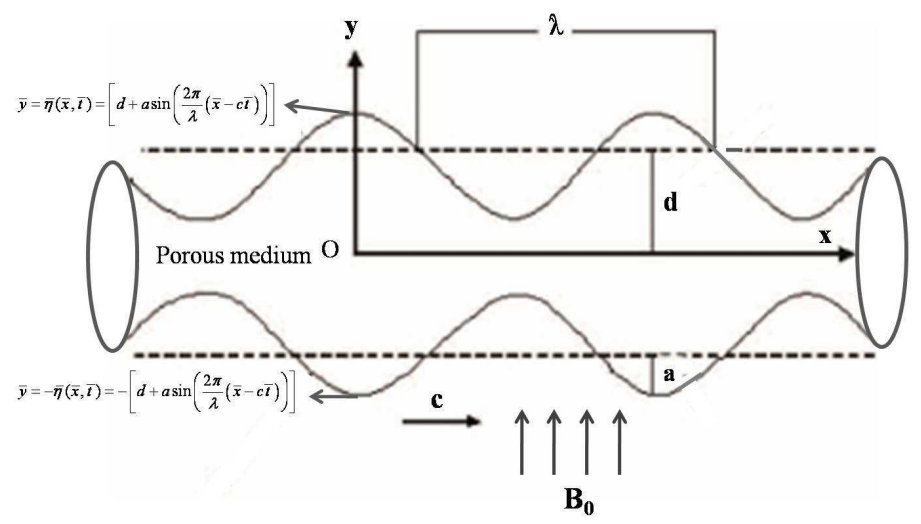

Geometry of 2- dimensional peristaltic motion of channel walls

symmetrical 2-dimensional channel through porous medium in the presence of transverse magnetic field. The half width of the channel is $d$. The waves on the channel walls have speed $\mathrm{c}$, and amplitude phase a. The wave length of the channel is $\lambda$. We select Cartesian coordinates $(\mathrm{x}, \mathrm{y})$ with $\mathrm{x}$ being in the direction of wave propagation and y normal to it. $\bar{\eta}$ is the displacement of upper wall, $-\bar{\eta}$ the displacement of lower wall and $t$ the time. The geometry of the wall surface is described by

$$
Y= \pm \bar{\eta}= \pm\left[d+a \sin \frac{2 \pi}{\lambda}(\bar{X}-c \bar{t})\right]
$$

The equations describing two dimensional flow are: For fluid particles:

$$
\begin{gathered}
\frac{\partial \bar{u}}{\partial \bar{x}}+\frac{\partial \bar{v}}{\partial \bar{y}}=0 \\
\left(\frac{\partial \bar{u}}{\partial \bar{t}}+\bar{u} \frac{\partial \bar{u}}{\partial \bar{x}}+\bar{v} \frac{\partial \bar{u}}{\partial \bar{y}}\right)=-\frac{1}{\rho} \frac{\partial \bar{p}}{\partial \bar{x}}+\nu\left(\frac{\partial^{2} \bar{u}}{\partial \bar{x}^{2}}+\frac{\partial^{2} \bar{u}}{\partial \bar{y}^{2}}\right) \\
+\frac{K N_{0}}{\rho}\left(\overline{u_{p}}-\bar{u}\right)-\frac{\nu}{K_{1}} \bar{u}-\frac{\sigma B_{0}^{2}}{\rho} \bar{u} \\
\left(\frac{\partial \bar{v}}{\partial \bar{t}}+\bar{u} \frac{\partial \bar{v}}{\partial \bar{x}}+\bar{v} \frac{\partial \bar{v}}{\partial \bar{y}}\right)=-\frac{1}{\rho} \frac{\partial \bar{p}}{\partial \bar{y}}+\nu\left(\frac{\partial^{2} \bar{v}}{\partial \bar{x}^{2}}+\frac{\partial^{2} \bar{v}}{\partial \bar{y}^{2}}\right)
\end{gathered}
$$




$$
+\frac{K N_{0}}{\rho}\left(\overline{v_{p}}-\bar{v}\right)-\frac{\nu}{K_{1}} \bar{v}
$$

For dust particles

$$
\begin{gathered}
\frac{\partial \overline{u_{p}}}{\partial \bar{x}}+\frac{\partial \overline{v_{p}}}{\partial \bar{y}}=0 \\
\left(\frac{\partial \overline{u_{p}}}{\partial \bar{t}}+\overline{u_{p}} \frac{\partial \overline{u_{p}}}{\partial \bar{x}}+\overline{v_{p}} \frac{\partial \overline{u_{p}}}{\partial \bar{y}}\right)=\frac{K}{m}\left(\bar{u}-\overline{u_{p}}\right) \\
\left(\frac{\partial \overline{v_{p}}}{\partial \bar{t}}+\overline{u_{p}} \frac{\partial \overline{v_{p}}}{\partial \bar{x}}+\overline{v_{p}} \frac{\partial \overline{v_{p}}}{\partial \bar{y}}\right)=\frac{K}{m}\left(\bar{v}-\overline{v_{p}}\right)
\end{gathered}
$$

With the appropriate boundary conditions $\bar{u}=0, \overline{u_{p}}=0 \quad$ at $\quad \bar{y}= \pm \bar{\eta}$ Where $\bar{u}, \bar{v}$ is the velocity of the fluid particles and $\bar{u}_{p}, \overline{v_{p}}$ is the velocity of the dust particles, $\bar{p}$ - Fluid pressure, $B_{0}$ - Transverse magnetic field, $\sigma$ - Coefficient of electric conductivity, $\rho$ - Density of the fluid, $\nu$ - Kinematic coefficient of the viscosity of fluid, $\mathrm{K}$ - Resistance coefficient for the dust particles(a constant), $K_{1}$ - Permeability of the porous medium, m - Mass of the solid particles. The governing equation of motion of the flexible wall may be expressed as

$$
L(\bar{\eta})=\bar{p}-\overline{p_{0}}
$$

where $\mathrm{L}$ is an operator that is used to represent the motion of the stretched membrane with damping forces such that

$$
L=-T \frac{\partial^{2}}{\partial \bar{x}^{2}}+m^{\prime} \frac{\partial^{2}}{\partial \bar{t}^{2}}+C \frac{\partial}{\partial \bar{t}}
$$

T-Tension in the membrane, m-Mass per unit area, $\mathrm{C}$ - Coefficient of viscous damping forces and $\overline{p_{0}}$-Pressure on the outside of the wall due to tension in the muscles. With the appropriate boundary conditions,From equation (3)

$$
\begin{aligned}
\frac{\partial}{\bar{x}}(L(\bar{\eta}))=\frac{\partial \bar{p}}{\partial \bar{x}}=\rho \nu\left(\frac{\partial^{2} \bar{u}}{\partial \bar{x}^{2}}\right. & \left.+\frac{\partial^{2} \bar{u}}{\partial \bar{y}^{2}}\right)-\rho\left(\frac{\partial \bar{u}}{\partial \bar{t}}+\bar{u} \frac{\partial \bar{u}}{\partial \bar{x}}+\bar{v} \frac{\partial \bar{u}}{\partial \bar{y}}\right) \\
& +\frac{K N_{0}}{\rho}\left(\overline{u_{p}}-\bar{u}\right)-\frac{\nu}{K_{1}} \bar{u}-\frac{\sigma B_{0}^{2}}{\rho} \bar{u}
\end{aligned}
$$

Introducing the stream function $\bar{\psi}, \bar{\phi}$ such that

$$
\bar{u}=\frac{\partial \bar{\psi}}{\partial \bar{y}}, \bar{v}=\frac{\partial \bar{\psi}}{\partial \bar{x}}, \overline{u_{p}}=\frac{\partial \bar{\phi}}{\partial \bar{y}}, \overline{v_{p}}=\frac{\partial \bar{\phi}}{\partial \bar{x}}
$$


and the following non-dimensional variables

$$
x=\frac{\bar{x}}{\lambda}, y=\frac{\bar{y}}{d}, \eta=\frac{\bar{\eta}}{d}, t=\frac{\nu \bar{t}}{\lambda}, \psi=\frac{\bar{\psi}}{\nu}, \phi=\frac{\bar{\phi}}{K d^{2} / m},
$$

into equations $(2)$ - (7) we obtain the following equations (after eliminating the pressure term)

$$
\begin{gathered}
\delta\left[\frac{\partial}{\partial t}\left(\nabla_{1}^{2} \psi\right)+\frac{\partial \psi}{\partial y} \frac{\partial}{\partial x}\left(\nabla_{1}^{2} \psi\right)-\frac{\partial \psi}{\partial x} \frac{\partial}{\partial y}\left(\nabla_{1}^{2} \psi\right)\right] \\
=\nabla_{1}^{4} \psi+P\left[\frac{1}{R} \nabla_{2}^{1} \phi-\nabla_{2}^{1} \psi\right]-\frac{1}{k_{0}} \nabla_{1}^{2} \psi-M^{2} \frac{\partial^{2} \psi}{\partial y^{2}} \\
\delta\left[R \frac{\partial}{\partial t}\left(\nabla_{1}^{2} \phi\right)+\frac{\partial \phi}{\partial y} \frac{\partial}{\partial x}\left(\nabla_{1}^{2} \phi\right)-\frac{\partial \phi}{\partial x} \frac{\partial}{\partial y}\left(\nabla_{1}^{2} \phi\right)\right]=R \nabla_{1}^{2} \psi-\nabla_{1}^{2} \phi
\end{gathered}
$$

where

$$
\nabla_{1}^{2}=\left(\frac{\partial^{2}}{\partial X^{2}}+\frac{\partial^{2}}{\partial Y^{2}}\right), \epsilon=\frac{a}{d}, \delta=\frac{d}{\lambda}
$$

$R=\frac{\nu m}{K d^{2}} \quad$ and $\quad P=\frac{K N_{0} d^{2}}{\nu p} \quad$ are non-dimensional parameters and $k_{0}=\frac{K_{1}}{d^{2}}$ is the coefficient of permeability of porous medium, $M^{2}=\frac{B_{0} d \bar{\sigma}}{\bar{\mu}}$ is the Hartmann number The boundary conditions in the non dimensional form is

$$
\begin{gathered}
\frac{\partial \psi}{\partial y}=\frac{\partial \phi}{\partial y}=0 \quad \text { at } \quad y= \pm \eta= \pm[1+\epsilon \sin 2 \pi(x-t)] \\
\nabla_{1}^{2} \frac{\partial \psi}{\partial y}-\delta\left[\frac{\partial}{\partial t} \frac{\partial \psi}{\partial y}+\frac{\partial \psi}{\partial y} \frac{\partial}{\partial x} \frac{\partial \psi}{\partial y}-\frac{\partial \psi}{\partial x} \frac{\partial}{\partial y} \frac{\partial \psi}{\partial y}\right]+P\left[\frac{1}{R} \frac{\partial \phi}{\partial y}-\frac{\partial \psi}{\partial y}\right] \\
-\left(\frac{1}{k_{0}}+M^{2}\right) \frac{\partial \psi}{\partial y}=\left(E_{1} \frac{\partial^{3}}{\partial x^{3}}+E_{2} \frac{\partial^{3}}{\partial x \partial t^{2}}+E_{3} \frac{\partial^{2}}{\partial x \partial t}+\right) \eta
\end{gathered}
$$

at $y= \pm \eta= \pm[1+\epsilon \sin 2 \pi(x-t)]$ where

$$
\begin{gathered}
E_{1}=\frac{T d^{4}}{\nu \rho}-\text { MembraneTensionParameter, } \\
E_{2}=\frac{m^{\prime} d^{2}}{\lambda^{3} \rho}-\text { MasscharactarisingParameter } \\
E_{3}=\frac{C d^{3}}{\lambda^{3} \nu \rho}-\text { TheDampingTensionParameter }
\end{gathered}
$$




\section{Solution Procedure}

Assuming the parameter $\delta$ is very small, the stream functions $\psi$ and $\phi$ may be expanded in power series of $\delta$ as

$$
\psi=\psi_{0}+\delta \psi_{1}+\delta^{2} \psi_{2}+\ldots \ldots, \phi=\phi_{0}+\delta \phi_{1}+\delta^{2} \phi_{2}+\ldots \ldots
$$

Substituting (16) in equations (11) - (15) and collecting the coefficients of various powers of $\delta$ on both sides, we obtain the following sets of coupled linear differential equations for $\psi_{0}, \phi_{0}$ and $\psi_{1}, \phi_{1}$

Zeroth order in $\delta$

$$
\begin{gathered}
\frac{\partial^{4} \psi_{0}}{\partial y^{4}}+P\left[\frac{1}{R} \frac{\partial^{2} \phi_{0}}{\partial y^{2}}-\frac{\partial^{2} \psi_{0}}{\partial y^{2}}\right]-L^{2} \frac{\partial^{2} \psi_{0}}{\partial y^{2}}=0 \\
R \frac{\partial^{2} \psi_{0}}{\partial y^{2}}-\frac{\partial^{2} \phi_{0}}{\partial y^{2}}=0
\end{gathered}
$$

and the corresponding boundary conditions at $y= \pm \eta \quad$ are

$$
\begin{gathered}
\frac{\partial \psi_{0}}{\partial y}=\frac{\partial \phi_{0}}{\partial y}=0 \\
\frac{\partial^{3} \psi_{0}}{\partial y^{3}}+P\left[\frac{1}{R} \frac{\partial \phi_{0}}{\partial y}-\frac{\partial \psi_{0}}{\partial y}\right]-L^{2} \frac{\partial \psi_{0}}{\partial y} \\
=\left(E_{1} \frac{\partial^{3}}{\partial x^{3}}+E_{2} \frac{\partial^{3}}{\partial x \partial t^{2}}+E_{3} \frac{\partial^{2}}{\partial x \partial t}+\right) \eta
\end{gathered}
$$

First order in $\delta$

$$
\begin{gathered}
\frac{\partial^{4} \psi_{1}}{\partial y^{4}}+P\left[\frac{1}{R} \frac{\partial^{2} \phi_{1}}{\partial y^{2}}-\frac{\partial^{2} \psi_{1}}{\partial y^{2}}\right]-L^{2} \frac{\partial^{2} \psi_{1}}{\partial y^{2}} \\
=\left(\frac{\partial^{3} \psi_{0}}{\partial t \partial y^{2}}+\frac{\partial \psi_{0}}{\partial y} \frac{\partial^{3} \psi_{0}}{\partial x \partial y^{2}}-\frac{\partial \psi_{0}}{\partial x} \frac{\partial^{3} \psi_{0}}{\partial y^{3}}\right) \\
R \frac{\partial^{2} \psi_{1}}{\partial y^{2}}-\frac{\partial^{2} \phi_{1}}{\partial y^{2}}=R \frac{\partial^{3} \phi_{0}}{\partial t \partial y^{2}}+\frac{\partial \phi_{0}}{\partial y} \frac{\partial^{3} \phi_{0}}{\partial x \partial y^{2}}-\frac{\partial \phi_{0}}{\partial x} \frac{\partial^{3} \phi_{0}}{\partial y^{3}}
\end{gathered}
$$

and the corresponding boundary conditions at $y= \pm \eta$ are

$$
\frac{\partial \psi_{1}}{\partial y}=\frac{\partial \phi_{1}}{\partial y}=0
$$




$$
\begin{array}{r}
\frac{\partial^{3} \psi_{1}}{\partial y^{3}}+P\left[\frac{1}{R} \frac{\partial \phi_{1}}{\partial y}-\frac{\partial \psi_{1}}{\partial y}\right]-L^{2} \frac{\partial \psi_{1}}{\partial y} \\
=\frac{\partial^{2} \psi_{0}}{\partial t \partial y}+\frac{\partial \psi_{0}}{\partial y} \frac{\partial^{2} \psi_{0}}{\partial x \partial}-\frac{\partial \psi_{0}}{\partial x} \frac{\partial^{2} \psi_{0}}{\partial y^{2}}
\end{array}
$$

where

$$
L^{2}=\frac{1}{k_{0}}+M^{2}
$$

The solutions of equations (17) and (18), subject to the boundary conditions (19) and (20), are

$$
\begin{gathered}
\psi_{0}=\frac{A_{1} \sinh L y}{L^{3} \cosh L \eta}-\frac{A_{1}}{L^{2}} \\
\phi_{0}=R\left(\frac{A_{1} \sinh L y}{L^{3} \cosh L \eta}-\frac{A_{1}}{L^{2}}\right)
\end{gathered}
$$

where

$$
A_{1}=\epsilon\left[E_{3}(2 \pi)^{2} \sin 2 \pi(x-t)-\left(E_{1}+E_{2}\right)(2 \pi)^{3} \cos 2 \pi(x-t)\right]
$$

The solutions of equations (21) and (22), subject to the boundary conditions (23) and (24), are

$$
\begin{gathered}
\psi_{1}=T_{30} y+T_{31} \sinh L y+T_{12} y \cosh L y+T_{13} \sinh 2 L y+T_{14} y^{2} \sinh L y \\
\phi_{1}=T_{42} y+T_{38} \sinh L y+T_{39} y \cosh L y+T_{40} \sinh 2 L y+T_{41} y^{2} \sinh L y \\
S=\frac{N_{0} m}{\rho}=P R
\end{gathered}
$$

where $\mathrm{S}$ is mass concentration of dust particles, The axial velocities of the fluid $(\mathrm{u})$ and the dust particle $\left(u_{p}\right)$ are given by

$$
\begin{aligned}
& u=\frac{A_{1}}{L^{2}}\left(\frac{\cosh L y}{\cosh L \eta}-1\right)+\delta\left(T_{30}+T_{32} \cosh L y+2 T_{13} \cosh 2 L y\right) \\
& +\delta\left(T_{14} L y^{2} \cosh L y+T_{33} y \sinh L y\right)
\end{aligned}
$$




$$
\begin{array}{r}
u_{p}=\frac{A_{1} R}{L^{2}}\left(\frac{\cosh L y}{\cosh L \eta}-1\right)+\delta\left(T_{42}+\left(T_{38} L+T_{39}\right) \cosh L y\right) \\
+\delta\left(2 T_{40} L \cosh 2 L y+\left(T_{39} L+2 T_{41}\right) y \sinh L y+T_{41} L y^{2} \cosh L y\right)
\end{array}
$$

\section{Rusults and Discussion}

In this section, the behavior of various parameters involved in the expressions of axial velocity $u=\psi_{0}+\delta \psi_{1}$ and steam function $\psi$ are provided. In particular the variations of elastic tension in the membrane $E_{1}$, the mass per unit area $E_{2}$, the coefficient of viscous damping $E_{3}$, mass characterizing parameter S, Hartmann number $\mathrm{M}$ and permeability of the porous medium $k_{0}$ are observed. Fig. 1 shows the behavior of parameters which is involved in velocity profile. Fig. 1.a and 1.b depict that the velocity profile increases as the elastic tension in the membrane and the mass per unit area increases. Because of the elasticity of the walls, there is less resistance to the flow and so the velocity increases as noted by (17). Fig. 1.c shows that $u$ increases by increasing $E_{3}$. Figs. 1.d and 1.e show that the velocity decreases with an increase in mass characterizing parameter and Hartmann number. Fig. 1.f depicts that the velocity increases with an increase in the permeability of the porous medium $k_{0}$.

Trapping is an important phenomenon in peristaltic motion. In a wave frame, the streamline under particular conditions split to trap a bolus which moves as a whole with the speed of the peristaltic wave. The effects of various parameters like elastic tension in the membrane $E_{1}$, the mass per unit area $E_{2}$, the coefficient of viscous damping $E_{3}$, mass characterizing parameter S, Hartmann number $\mathrm{M}$ and permeability of the porous medium $k_{0}$ on trapping and reflux were numerically evaluated and the results are graphically presented in Fig. 2. Figs. 2.a and 2.b shows that the streamline got closer as the tension parameter $\left(E_{1}\right)$ increased and the phenomenon of trapping was observed. Observing figs. 2.a and 2.b it is significant to note that the trapping phenomenon became predominant and the area of the trapped bolus increased as the tension parameter increased. We observe from figs. 2.c and 2.d that the trapping phenomenon occurs for mass per unit area $\left(E_{2}\right)$ It can be seen from figs 2.c and 2.d that the trapped bolus increases as the mass per unit area $E_{2}$ increases. It can be seen figs. 2.e and 2.f that as damping force $E_{3}$ increased the area of the trapped bolus in some regions decreased (left side) and in some regions remained unchanged (right side). The effect of the mass characterizing parameter $(\mathrm{S})$ on the streamline pattern is shown in Figs. 2.g and 2.h. Change in 


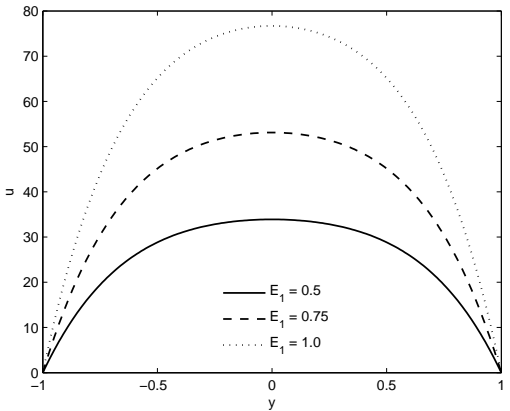

(a) Variation of $E_{1}$ on $\mathrm{u}$ when $k_{0}$ $=1, \mathrm{M}=0.2, \mathrm{~S}=0.2, E_{2}=0.5$, $E_{3}=0.6$.

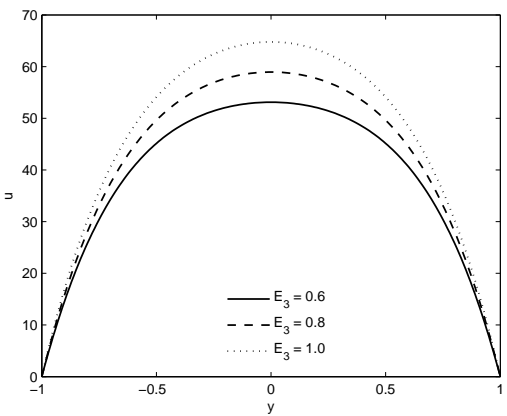

(c) Variation of $E_{3}$ on $\mathrm{u}$ when $k_{0}$ $=1, \mathrm{M}=0.2, \mathrm{~S}=0.2, E_{1}=0.75$, $E_{2}=0.5$.

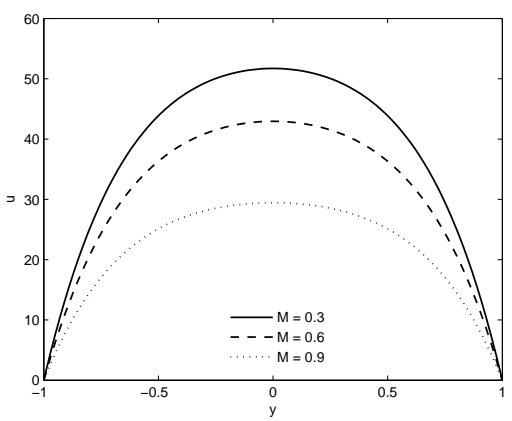

(e) Variation of $\mathrm{M}$ on $\mathrm{u}$ when $k_{0}$ $=1, \mathrm{~S}=0.2, E_{1}=0.75, E_{2}=$ $0.5, E_{3}=0.6$.

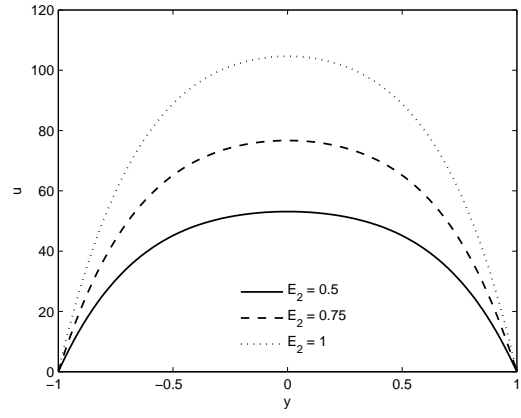

(b) Variation of $E_{2}$ on $\mathrm{u}$ when $k_{0}$ $=1, \mathrm{M}=0.2, \mathrm{~S}=0.2, E_{1}=0.75$, $E_{3}=0.6$.

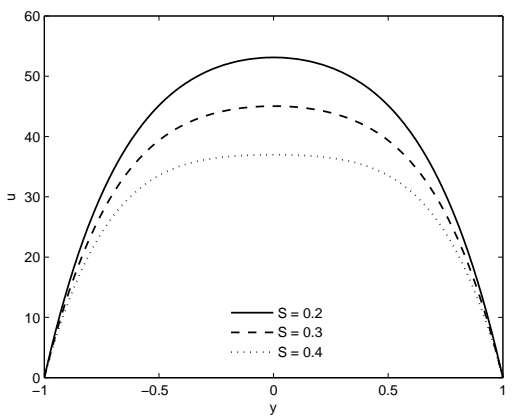

(d) Variation of $\mathrm{S}$ on $\mathrm{u}$ when $k_{0}$ $=1, \mathrm{M}=0.2, E_{1}=0.75, E_{2}=$ $0.5, E_{3}=0.6$.

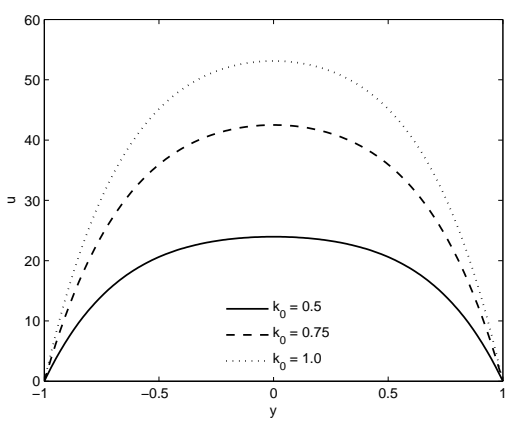

(f) Variation of $k_{0}$ on $\mathrm{u}$ when $\mathrm{S}$ $=0.2, \mathrm{M}=0.2, E_{1}=0.75, E_{2}=$ $0.5, E_{3}=0.6$.

Figure 1: velocity profile of the fluid when $\epsilon=0.2, \delta=0.2$. 


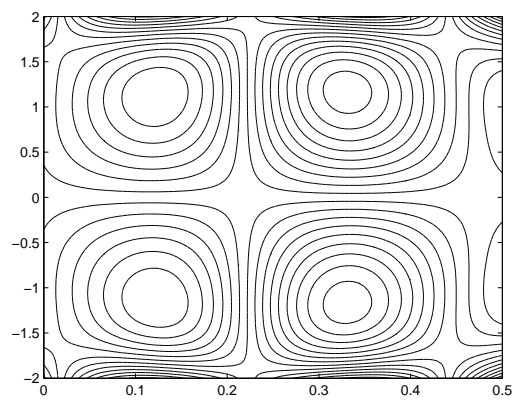

(a) $E_{1}=0.1$

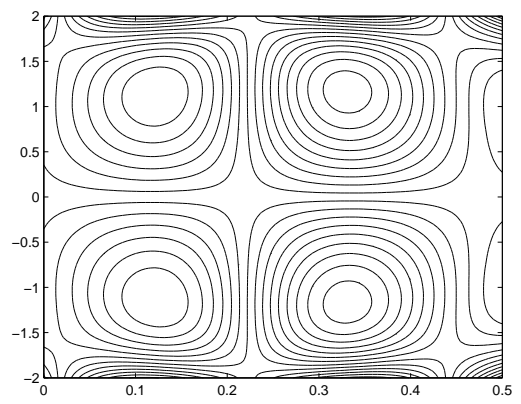

(c) $E_{2}=0.1$

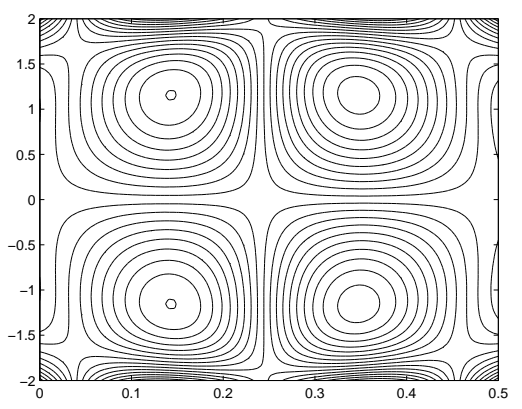

(e) $E_{3}=0.1$

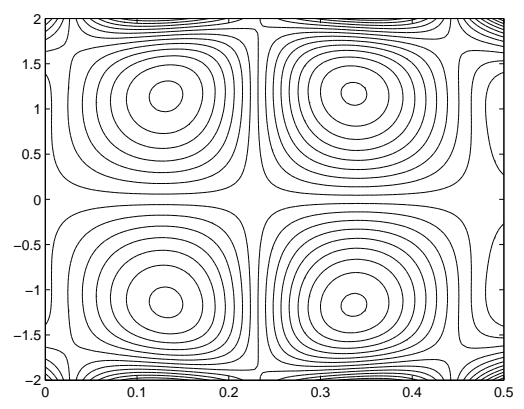

(b) $E_{1}=0.5$

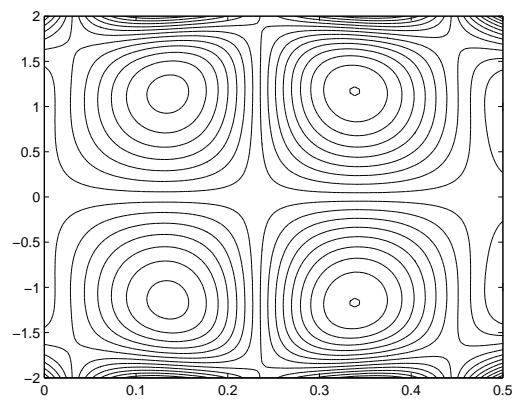

(d) $E_{2}=0.5$

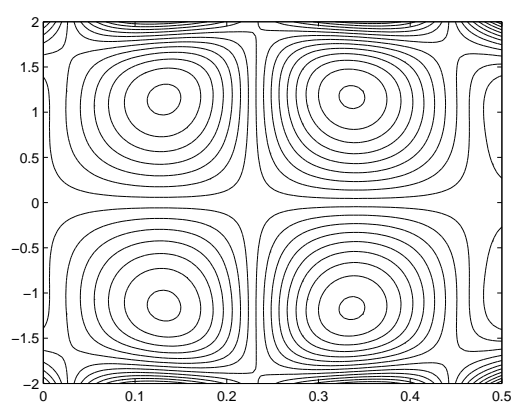

(f) $E_{3}=0.75$ 


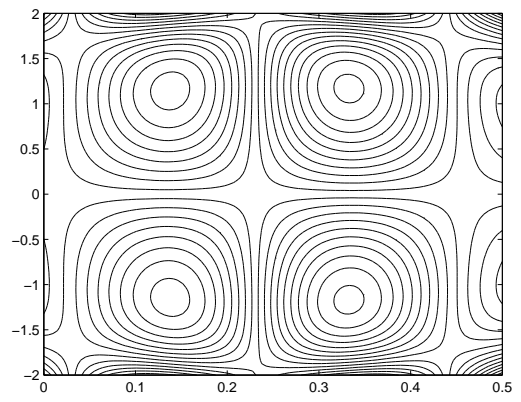

(a) $\mathrm{S}=0.1$

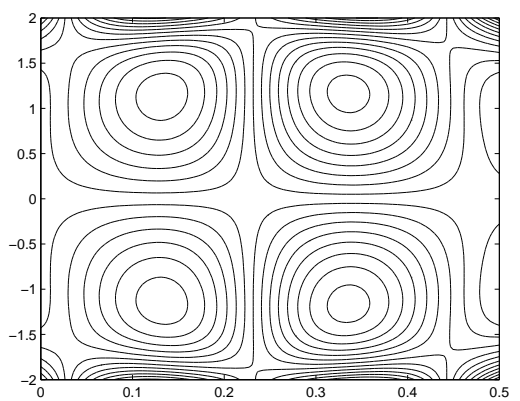

(c) $\mathrm{M}=0.1$

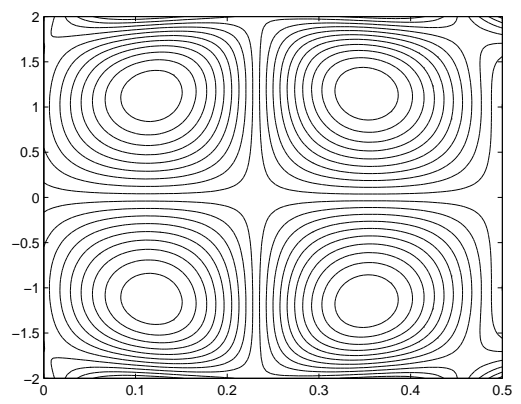

(e) $k_{0}=0.3$

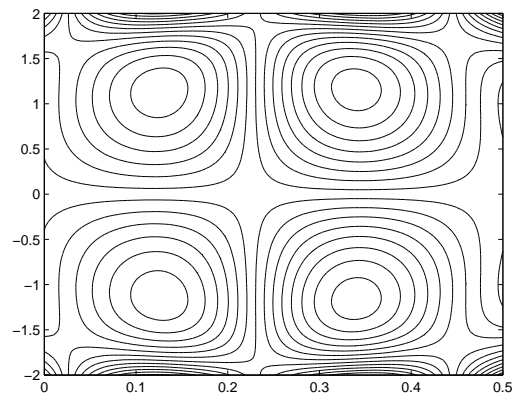

(b) $\mathrm{S}=0.75$

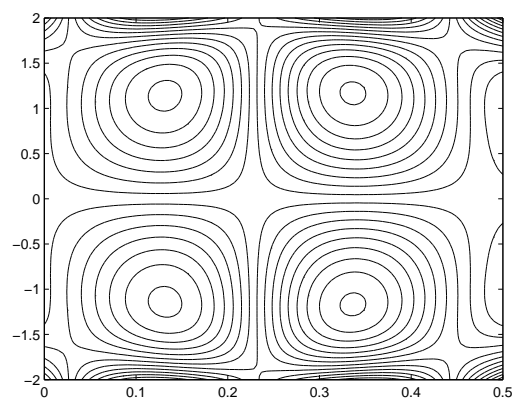

(d) $\mathrm{M}=0.2$

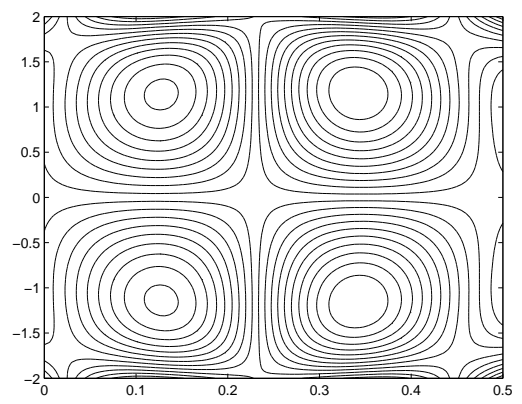

(f) $k_{0}=0.8$

Figure 3: Streamline pattern of the fluid particles for $\epsilon=0.2, \delta=0.2$, $E_{1}=0.5, E_{2}=0.75, E_{3}=0.75, \mathrm{~S}=0.5$ and $\mathrm{M}=0.2, k_{0}=1$ 
the character of the streamlines with the variation in the mass concentration of dust particles (S) was significant. The trapping was observed and the area of the trapped bolus decreases as increase of mass concentration of the dust particle S.

Figs. 2.i and 2.j shows the influence of Hartmann number M on trapping. It shows that the size of the left trapped bolus increases on both sides with increase in M. The influence of porous permeability parameter $k_{0}$ on the trapping is analyzed in figs $2 . \mathrm{k}$ and 2.l. It showed that the volume of the trapped bolus is unchanged with increasing permeability parameter $k_{0}$.

\section{Concluding Remarks}

The present study deals with the effect of wall properties on MHD peristaltic flow of a dusty fluid through a porous medium channel. We obtained the analytical solution of the problem under long wavelength and low Reynolds number assumptions. The results are analyzed for different values of parameters namely elastic tension in the membrane $E_{1}$, the mass per unit area $E_{2}$, the coefficient of viscous damping $E_{3}$, mass characterizing parameter S, Hartmann number M, permeability of the porous medium $k_{0}$. The key features of the present study are

1. The axial velocity increases as the elastic tension in the membrane and the mass per unit area increases. The velocity increases with an increase in the permeability of the porous medium $k_{0}$. the velocity decreases with an increase in mass characterizing parameter and Hartmann number.

2. The volume of the trapped bolus increases with increase in $E_{1}, E_{2}$ and $k_{0}$. Moreover, more trapped bolus appears with increase in $E_{1}$ and $E_{2}$.

3. The volume of the trapped bolus decreases with increase in S.

4. The volume of the left trapped bolus decreases with increase in $E_{3}$ and $\mathrm{M}$, whereas it has opposite behavior on the right hand side of the channel.

\section{Appendix}

$$
T_{1}=\frac{1}{L}\left(\frac{\cosh L \eta \frac{\partial A_{1}}{\partial t}-A_{1} L \sinh L \eta \frac{\partial \eta}{\partial t}}{(\cosh L \eta)^{2}}\right)
$$




$$
\begin{aligned}
& T_{2}=\frac{1}{L}\left(\frac{\cosh L \eta \frac{\partial A_{1}}{\partial x}-A_{1} L \sinh L \eta \frac{\partial \eta}{\partial x}}{(\cosh L \eta)^{2}}\right) \\
& T_{3}=\frac{T_{2} A_{1}}{2 L^{2} \cosh L \eta}, \quad T_{4}=-\frac{T_{2} A_{1}}{L^{2}}, \quad T_{5}=\frac{T_{2}}{L^{2}}, \\
& T_{6}=-\frac{\frac{\partial A_{1}}{\partial x}}{L^{2}}, \quad T_{7}=\frac{T_{5} A_{1}}{2 \cosh L \eta}, \quad T_{8}=\frac{T_{6} A_{1}}{\cosh L \eta} \\
& T_{9}=S_{1}\left(T_{1}+T_{4}\right), \quad T_{10}=S_{1}\left(T_{3}-T_{7}\right), \quad T_{11}=-S_{1} T_{8}, \\
& T_{12}=\frac{T_{9}}{2 L^{3}}-\frac{5 T_{11}}{4 L^{3}}, \quad T_{13}=\frac{T_{10}}{12 L^{4}}, \\
& T_{14}=\frac{T_{11}}{4 L_{3}}, \quad T_{17}=\frac{T_{1}}{L}, \quad T_{18}=-\frac{\frac{\partial A_{1}}{\partial t}}{L^{2}}, \\
& T_{19}=\frac{T_{2}}{L}, \quad T_{20}=T_{6}, \quad T_{21}=\frac{A_{1}}{L^{2} \cosh L \eta}, \quad T_{22}=-\frac{A_{1}}{L^{2}}, \\
& T_{23}=T_{21} T_{19}, \quad T_{24}=T_{20} T_{21}+T_{22} T_{19}, \quad T_{25}=T_{22} T_{20}, \\
& T_{26}=\frac{T_{5} A_{1}}{L \cosh L \eta}, \quad T_{27}=\frac{T_{6} A_{1}}{L \cosh L \eta}, \\
& T_{28}=T_{17}+T_{24}, \quad T_{29}=T_{18}+T_{25}, \quad T_{32}=T_{31} L+T_{12}, \quad T_{33}=T_{12} L+2 T_{14}, \\
& T_{30}=\frac{1}{L^{2}}\left(\left(2 T_{12} L^{2}+6 T_{14} L-T_{28}\right) \cosh L \eta+6 T_{13} L^{3} \cosh 2 L \eta\right) \\
& +\frac{1}{L^{2}}\left(\left(4 T_{14} L^{2}+T_{27}\right) \eta \sinh L \eta-T_{29}-T_{23}(\cosh L \eta)^{2}+T_{26}(\sinh L \eta)^{2}\right), \\
& T_{31}= \\
& -\frac{\left(T_{28}+\left(T_{12} L+2 T_{14}\right) \eta \sinh L \eta\right)+\left(T_{14} L \eta^{2}+T_{12}\right) \cosh L \eta+2 T_{13} L \cosh 2 L \eta}{L \cosh L \eta}, \\
& T_{34}=R\left(T_{31} L^{2}+2 T_{14} L+2 T_{14}\right)-R^{2} T_{1}-R^{2} T_{4}, T_{35}=R\left(T_{12} L^{2}+4 T_{14} L\right)+R^{2} T_{8}, \\
& T_{36}=4 R T_{13} L^{2}-R^{2} T_{3}+R^{2} T_{7}, \quad T_{37}=R T_{14} L^{2}, \\
& T_{38}=\frac{T_{34}}{L^{2}}-\frac{2 T_{35}}{L^{3}}+\frac{T_{37}}{L^{4}}, \quad T_{39}=\frac{T_{35}}{L^{2}}-\frac{2 T_{37}}{L^{3}}, \\
& T_{40}=\frac{T_{36}}{4 L^{2}}, \quad T_{41}=\frac{T_{37}}{L^{2}}, \\
& T_{42}=-\left(\left(T_{38} L+T_{39}+T_{41} L \eta^{2}\right) \cosh L \eta\right. \\
& \left.+\left(T_{39} L \eta+2 T_{41} \eta\right) \sinh L \eta+2 T_{40} L \cosh 2 L \eta\right) .
\end{aligned}
$$




\section{References}

[1] P.G.Saffman, the Stability of Laminar flow of a Dusty Gas J. Fluid Mech., 13 (1962), 122-128.

[2] T.W.Latham, Fluid motion in Peristaltic pump, MS thesis, Mit Cambridge, (1966).

[3] A.H.Shapiro, M.Y.Jaffrin and S.L.Weinberg, Peristaltic pumping with long wavelength at low Reynolds number, Journal of fluid mechanics, 37 (1969), 799-825.

[4] K.Tridib, Mittra and ShyamN.Prasad, On the influence of wall properties and Poiseuille flow in peristalsis, Journal of Biomechanics, 6 (1973), 681693.

[5] M. R. Kaimal, Peristaltic Pumping of a Newtonian Fluid With Particles Suspended in It at Low Reynolds Number Under Long Wavelength Approximations, Journal of Applied Mechanics, 45 (1978), 32-36.

[6] G. Radhakrishnama Charya, Pulsatile flow of a dusty fluid through a constricted channel, Zeitschrift fr angewandte Mathematik und Physik ZAMP, 29 (1978), 217-225.

[7] Kh.S.Mekheimer and T.H.Al-Arabi, Nonlinear peristaltic transport of MHD flow through a porous medium, IJMMS, 26 (2003), 16631682.

[8] P.Muthu, B.V.Rathish Kumar and Peeyush Chandra and J.Anziam, On the influence of wall properties in the peristaltic motion of micropolar fluid, ANZIAM J, 45 (2003), 245260.

[9] A.Mokhtar, Abd Elnaby, Mohamed H. Haroun, A new model for study the effect of wall properties on peristaltic transport of a viscous fluid, Communications in Nonlinear Science and Numerical Simulation, 13 (2008), 752762 .

[10] Mohamed H.Haroun, Effect of wall compliance on peristaltic transport of a Newtonian fluid in an asymmetric channel, Mathematical Problems in Engineering, Article ID 61475 (2006), 119.

[11] G. Radhakrishnamacharya, Ch. Srinivasulu, Influence of wall properties on peristaltic transport with heat transfer, C. R. Mecanique, 335 (2007), 369373. 
[12] P. Muthu, B.V. Rathish Kumar, Peeyush Chandra, Peristaltic motion of micropolar fluid in circular cylindrical tubes Effect of wall properties, $A p$ plied Mathematical Modelling, 32 (2008), 20192033.

[13] D. Srinivasacharya, G.Radhakrishnamacharya, CH.Srinivasulu, The Effects of Wall Properties on Peristaltic Transport of a Dusty Fluid, Turkish J. Eng. Env. Sci, 32 (2008), 357365.

[14] G. C. Sankad, G. Radhakrishnamacharya, Effect of wall properties on peristaltic transport of micropolar fluid in a non-uniform channel, Int. J. of Appl. Math and Mech, 6 (2010), 94-107.

[15] N. A. S. Afifia, S. R. Mahmoudb and H. M. Al-Isedec, Effect of Magnetic Field and Wall Properties on Peristaltic Motion of Micropolar Fluid, International Mathematical Forum, 6 (2011), 13451356.

[16] T. Hayat, S. Hina, Awatif A. Hendi, S. Asghar, Effect of wall properties on the peristaltic flow of a third grade fluid in a curved channel with heat and mass transfer, International Journal of Heat and Mass Transfer, 54 (2011), 51265136 .

[17] S. Hina, T. Hayat, S. Asghar, Awatif and A. Hendi, Influence of compliant walls on peristaltic motion with heat mass transfer and chemical reaction, International Journal of Heat and Mass Transfer, 55 (2012), 33863394.

[18] Nabil.T.Eldabe, kawther.A.Kamel, Galila.M.Abd-Allah and Shaimaa.F.Ramadan Peristaltic motion with heat and mass transfer of a dusty fluid through a horizontal porous channel under the effect of wall properties, IJRRAS, 15 (2013), 300-311.

[19] P. Srinivasa Rao, G. Bhanodaya Reddy, Effect of reflux on peristaltic motion in an asymmetric channel with partial slip and different wave forms, International journal of mechanical engineering (IJME), 3 (2014), 35-48.

[20] Dheia G. Salih, Al-Khafajy and Ahmed M. Abdulhadi, Effects of wall properties and heat transfer on the peristaltic transport of a Jeffrey fluid through porous medium channel, Mathematical Theory and Modeling , 4 (2014), pp. 88-99.

[21] S. Nadeem, Arshad Riaz, R. Ellahi, N. S. Akbar, Mathematical model for the peristaltic flow of Jeffrey fluid with nanoparticles phenomenon through a rectangular duct, Appl Nanosci, 4 (2014), 613-624. 
[22] S. Nadeem, Arshad Riaz and R. Ellahi, Peristaltic flow of viscous fluid in a rectangular duct with compliant walls, Computational Mathematics and Modeling, 25 (2014), 404- 415.

[23] G.C.Sankad, P.S.Nagathan, Asha Patil, M.Y.Dhange, Peristaltic Transport of a Herschel-Bulkley Fluid in a Non-Uniform Channel with Wall Effects, International Journal of Engineering Science and Innovative Technology, 3 (2014), 669-678. 
